\title{
158 Zystische Fibrose (Mukoviszidose)
}

\author{
S. Schmitt-Grohé, M. J. Lentze, J. Henker
}

\subsection{Genetische Grundlagen und Pathophysiologie}

S. Schmitt-Grohé, M. J. Lentze

Genetik Der genetische Defekt bei Mukoviszidose liegt auf dem langen Arm von Chromosom 7 mit autosomal-rezessivem Erbgang. Das CF-Gen umfasst 250.000 Basenpaare, die ein 1480 Aminosäuren enthaltendes Protein, das den „cystic fibrosis transmembrane regulator" (CFTR) kodiert. Inzwischen sind über 1900 Mutationen ( $\triangleright$ www.genet.sickkids.on.ca/cftr) bekannt. Die CFTR-Mutationen werden in 5 Klassen eingeteilt (• Abb. 158.1):

- Klasse I: CFTR wird überhaupt nicht synthetisiert,

- Klasse II: aufgrund fehlerhafter Faltung im endoplasmatischen Retikulum findet kein Processing von funktionstüchtigem CFTR-Protein statt,

- Klasse III: gestörte Aktivierung und Regulation des CFTRProteins,

- Klasse IV: gestörter Ionenfluss,

- Klasse V: eine reduzierte Menge an intaktem Protein liegt vor.

Die häufigste Mutation in Deutschland ist die Klasse-II-Mutation $\Delta$ F508 mit einer Prävalenz (je nach geographischer Region) von $62-77 \%$, ähnliche Zahlen gelten für Mitteleuropa und die europäisch-stämmigen Einwohner der USA. Bei $\Delta$ F508-Homozygoten findet sich eine Assoziation zur Pankreasinsuffizienz, aber keine zum pulmonalen Verlauf. Der progressive Untergang von Lungengewebe infolge von Inflammation und Infektion ist entscheidend für die Prognose der Mukoviszidose. Ausreichend gepowerte Studien aus den USA haben Mutationen im Transforming-growth-factor- $\beta 1-G e n$ (-509 und Codon-10-CC-Polymorphismen) als wichtigste Modifiergene für den pulmonalen Verlauf identifizieren können.

Pathophysiologie Pathophysiologisch führt der Gendefekt zum Mangel an funktionstüchtigem CFTR-Protein. CFTR ist ein cAMPabhängiges Transmembranprotein mit Expression an apikalen Membranen der Epithelzellen von exkretorischen Drüsen. Folgen des Mangels an funktionstüchtigem CFTR-Protein sind eine Hyposekretion von Chlorid und eine Aktivierung des epithelialen Natriumkanals. Konsekutiv wird eine Hyperabsorption von Natrium in die Zelle diskutiert und eine Volumendepletion des Flüssigkeitsfilms auf dem Epithel (z. B. „airway surface liquid“, ASL). Auf dem Atemwegsepithel führt dieses zu einem Verlust der Zilienmotilität und einer verminderten mukoziliären Clearance. Neben dem CFTR-Protein wird der mukoziliäre Transport noch über andere Mechanismen geregelt: Der P2Y-Rezeptor wird über ATP unabhängig von CFTRProtein aktiviert und induziert eine Chloridsekretion durch einen alternativen Chloridkanal.

Zusammenfassend führt diese Fehlfunktion des CFTR-Chloridkanals an der Oberfläche der sekretorischen Epithelzellen von exokrinen Drüsen zu den in • Abb. 158.2 dargestellten klinischen Manifestationen.

Epidemiologie Die Inzidenz der Mukoviszidose in Deutschland liegt bei 1:3300. Die Diagnosestellung erfolgt über Schweißtest, molekulargenetische Analyse der Mutationen und Messung der rektalen oder nasalen Potenzialdifferenz. Ein flächendeckendes Neugeborenenscreening in Deutschland besteht bisher nicht. Ein positives Screening erfordert die Bestätigung durch mindestens einen positiven Schweißtest und ggf. weitere Diagnostik (s. oben).

Die mittlere Überlebenszeit der Mukoviszidose liegt etwas unter 42 Jahren in Deutschland.

\subsection{Pulmonale Manifestationen}

\section{S. Schmitt-Grohé, M. J. Lentze}

Die Lungenerkrankung bei Mukoviszidose lässt sich durch eine chronische Atemwegsinfektion mit Progression zu Bronchiektasen, Belüftungsstörungen bis hin zur respiratorischen Insuffizienz mit Hypoxie und Hyperkapnie charakterisieren.

Inflammation und Infektion Bei Geburt scheinen die Lungen unbeeinträchtigt, rasch lassen sich auch bei klinisch gesund wirkenden Säuglingen in der bronchoalveolären Lavage Zeichen der Inflammation und Infektion mit neutrophilen Granulozyten nachweisen. Im Alter von weniger als 4 Monaten bei Säuglingen lassen sich schon bei $80 \%$ Auffälligkeiten in der CT der Lunge nachweisen (klinische Krankheitszeichen nur bei 15,8\%).

Während in den ersten Lebensjahren Bakterien wie Haemophilus influenzae und Staphylococcus aureus im Vordergrund stehen, findet sich im 10. Lebensjahr bei mehr als $30 \%$ Pseudomonas aeruginosa. Der Hauptgrund für die Affinität von P. aeruginosa für die CF-Lunge wird im Mangel an ASL mit der Folge einer gestörten Zilienfunktion bzw. einer gestörten mukoziliären Clearance gesehen. Auch spielen
- Abb. 158.1 CF-Mutationen und ihre Mechanismen: Einteilung in 5 Klassen (Klasse $1-V$ ) und ihre funktionellen Auswirkungen auf das CFTR-Protein

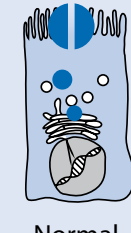

Normal

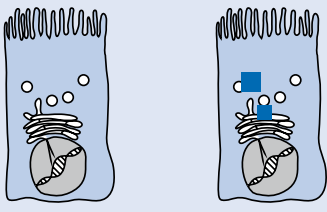

II

Mis-Sense

AS-Deletion $\Delta \mathrm{F} 508$

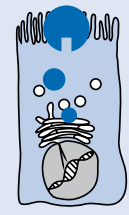

III

Mis-Sense G551

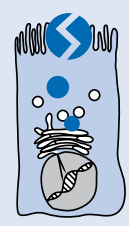

IV Mis-Sense $\mathrm{R} 117 \mathrm{H}$

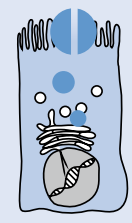

$\mathrm{V}$ Mis-Sense A455E Alternatives Splicen3849+ $10 \mathrm{kbC} \rightarrow \mathrm{T}$ 


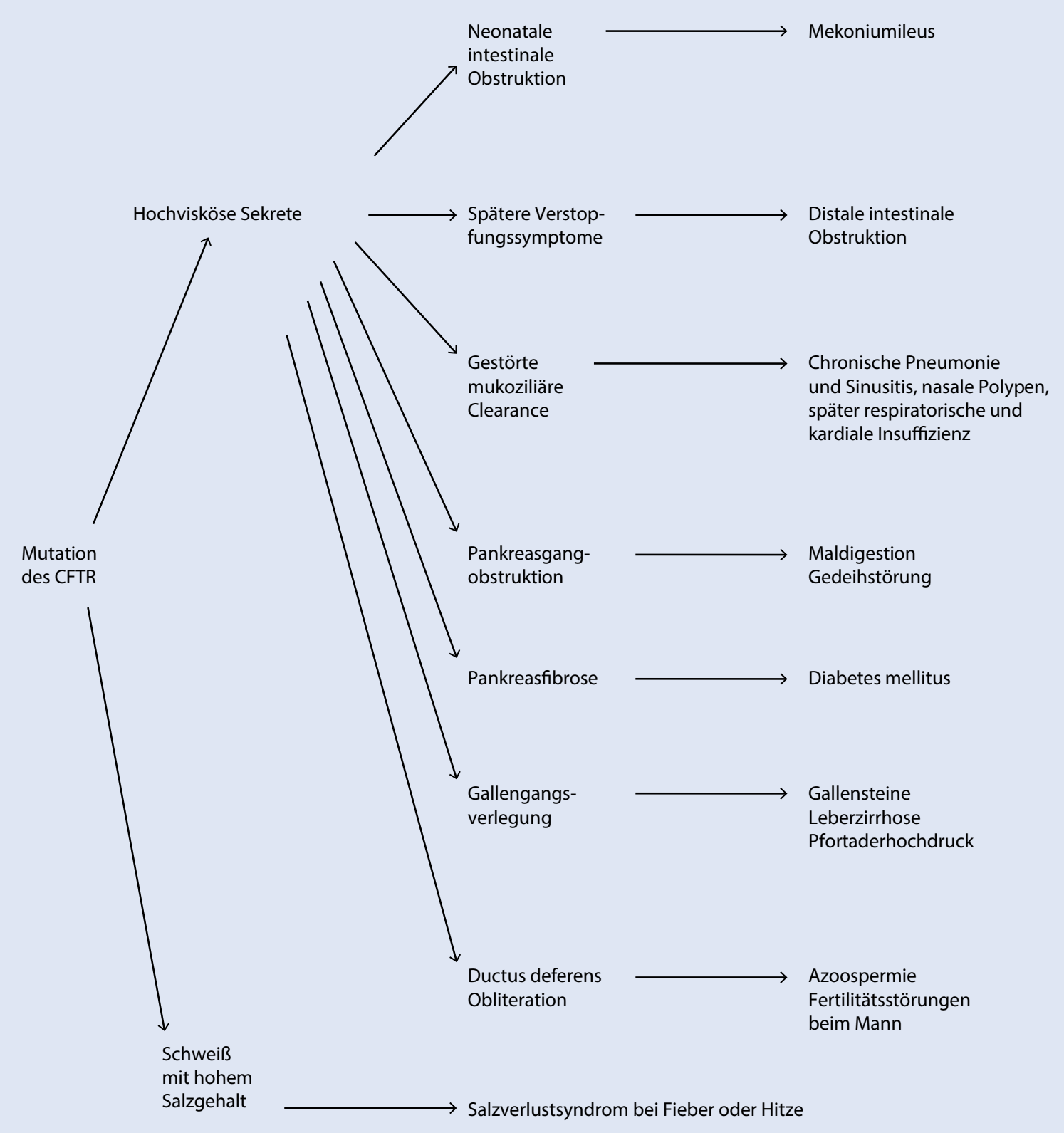

- Abb. 158.2 Pathophysiologie der Mukoviszidose

CF-spezifische Imbalancen der „innate immunity“ wie ein Mangel an intaktem Muzin im CF-Schleim eine Rolle. Der Sauerstoffmangel im CF-Sputum triggert den Wechsel vom nichtmukoiden zum mukoiden Phänotyp. Probleme des mukoiden Phänotyps sind die Resistenz gegen Abwehrmechanismen des Wirtsorganismus. Die gebildeten Biofilme sind schwer mit Antibiotika zu beheben.

Im Rahmen der persistierenden Infektion werden vermehrt chemotaktische Zytokine (u. a Interleukin-8) sezerniert mit konsekutiver Rekrutierung von neutrophilen Granulozyten. Es kommt zur Freisetzung von Toxinen und Elastasen, welche z. B. die Interleukin8-Rezeptoren zerstören, mit der Folge von noch mehr ungebundenem Interleukin-8 in der Lunge.

Bakterielle Exotoxine und Zerfallsprodukte von angegriffenen Neutrophilen unterhalten den weiteren Influx von Neutrophilen in die Lunge. Die Freisetzung von DNA von gealterten Neutrophilen steigert die Viskosität des Sputums.
Weitere für den pulmonalen Verlauf relevante Bakterien sind Burkholderia cepacia, Stenotrophomonas maltophilia, methicillinresistente Staphylokokken (MRSA) und atypische Mykobakterien.

Klinische Symptome und Verlauf Kinder mit chronisch persistierendem Husten, obstruktiver Bronchitis oder rezidivierenden Pneumonien sind verdächtig auf eine pulmonale Verlaufsform der Mukoviszidose. Patienten mit einer milden CF präsentieren sich zum Teil auch nur mit einer chronischen Sinusitis oder nasalen Polypen.

Die Auskultation spiegelt oben genannte Symptome wider: Rasselgeräusche, initial feinblasig bei Pneumonie, im fortgeschrittenen Stadium grobblasig bei Bronchiektasen. Bei obstruktiver Symptomatik Giemen und Brummen. Im Zuge der fortschreitenden Obstruktion und Überblähung können die Patienten einen Fassthorax entwickeln. Als Ausdruck der chronischen Obstruktion finden sich Uhrglasnägel und Trommelschlägelfinger. 
- Abb. 158.3a-c Radiologische und CT-Veränderungen. a Endstage-Lunge. a.-p.-Aufnahme: peribronchiale Verdickungen (kleiner Pfeil), Ringschatten (dicke Pfeile) als Hinweis auf Bullae, Pneumatozele oder Abszessbildung; Seitenbild: vergrößerter Retrosternalraum (Überblähung und Krümmung des Sternums), längere Pfeile markiertes Interlobium. Auf beiden Abbildungen deutliche Wabenbildungen (17 Jahre alte weibliche Patientin). b Atelektasenbildung rechter Oberlappenbronchus, wochenlang therapierefraktär trotz mehrmaliger bronchoskopischer Absaugung (16 Jahre alte weibliche Patientin). c HRCT der Lungen: große Bullenbildung rechts, auf der Nativröntgenaufnahme nicht nachweisbar (15 Jahre alter männlicher Patient)
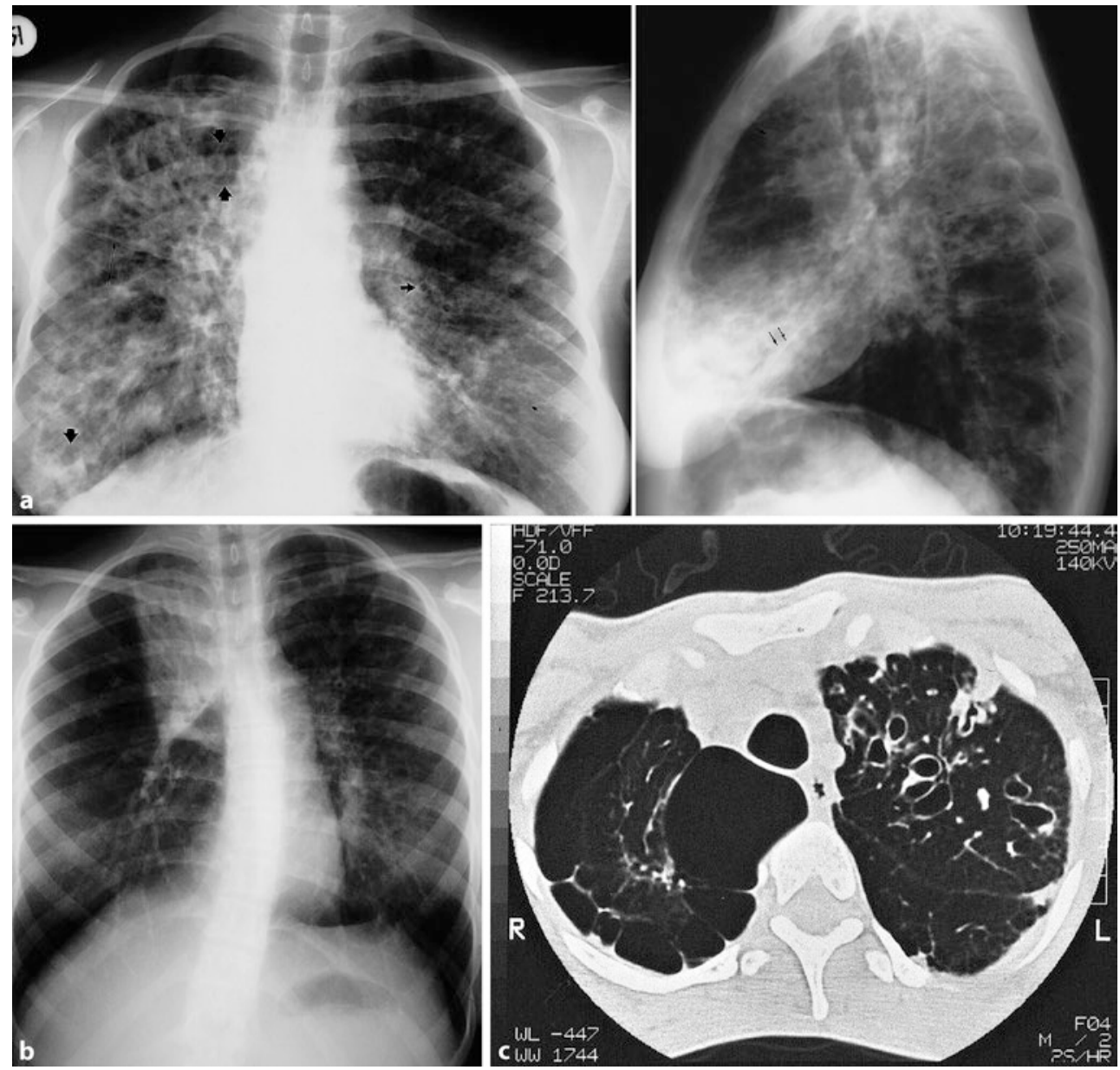

Zeichen der pulmonalen Exazerbation sind vermehrter Husten, Veränderung der Sputummenge oder -farbe, signifikanter Gewichtsverlust, zunehmende Abgeschlagenheit und Krankheitsgefühl, Abfall der Lungenfunktion um mehr als $10 \%$ und/oder Zunahme der radiologischen Veränderungen, zunehmende Atemnot.

Diagnose Folgende Diagnostik sollte in regelmäßigen Abständen und bei einem Verdacht auf pulmonale Exazerbation durchgeführt werden:

Mikrobiologische Diagnostik Mikrobiologische Diagnostik (Rachenabstriche oder Sputum) sollte bei chronisch Besiedelten mindestens alle 3 Monate erfolgen. Bei Patienten mit bisher negativen Befunden sind 4- bis 8- wöchentliche Kontrollen zu empfehlen. Sollte der Patient nicht in der Lage sein, Sputum abzuhusten, sollte neben tiefen Rachenabstrichen (negativ prädiktiver Wert $95 \%$, positiv prädiktiver Wert $44 \%$ ) auch induziertes Sputum gewonnen werden. Die Gewinnung erfolgt durch Abhusten nach Inhalation von 3-7\%iger NaCl-Lösung (um einen Bronchospasmus zu verhindern, sollte vorher protektiv mit Sultanol inhaliert werden). Nasale Abstriche oder Lavage sind zu diskutieren. Weitere diagnostische Bausteine sind Pseudomonas-Antikörper bei klinischem Verdacht und mangelndem mikrobiologischem Nachweis. Invasive Diagnostik wie die Gewinnung bronchoalveolärer Lavage sollte pulmonalen Exazerbationen, die anderweitig diagnostisch nicht zu klären sind, vorbehalten sein. Neben den mikrobiologischen Befunden sind die Lungenfunktionsparameter wichtig für den Verlauf.

\section{Lungenfunktion}

Säuglings- und Vorschulalter Vormals wurde das Vorschulalter als die unauffälligen Jahre in Bezug auf die spirometrisch gemessene Lungenfunktion gewertet. Neuere Methoden stellen diese Sicht infrage: Bereits Säuglinge haben bereits eine schlechtere Lungenfunktion (gemessen mit der Raised-volume-Technik) in Bezug auf die Forced expiratory flows and volumes (FEFV) als gesunde Gleichaltrige. Dieses Defizit (gemessen mittels Bodyplethysmografie und Spirometrie) ist auch im Vorschulalter nachweisbar. Negative Einflussfaktoren auf die Lungenfunktion sind die neutrophilengetriggerte Inflammation der Atemwege und Infektionen mit S. aureus und $\mathrm{P}$. aeruginosa.

Ab dem 4-5. Lebensjahr sollte eine routinemäßige Lungenfunktionsprüfung mittels Bodyplethysmografie und Spirometrie erfolgen.

Patienten jenseits des Vorschulalters Einer der ersten Auffälligkeiten ist eine Überblähung gemessen am vermehrten intrathorakalen Gasvolumen (ITGV) oder Residualvolumen (RV). Erste Probleme in der Spirometrie betreffen die kleinen Atemwege: Die Fluss-Volumen-Kurve zeigt verminderte Werte für den Maximum expiratory flow (MEF) bei 25, 50-75\% (MEF 25-75\%) der forcierten Vitalkapazität (FVC).

Der wichtigste Parameter für den klinischen Verlauf bzw. die Prognose ist die Einsekundenkapazität (FEV1). In der Regel geht man von 2-3\% Verlust an Lungenfunktion pro Jahr aus. Pulmonale Exazerbationen führen bei einem Viertel der Patienten zu einem persistierenden Verlust an Lungenfunktion. Sollte die Lungenfunktion im Schulalter unauffällig sein, darf deswegen nicht auf eine entzündungsfreie Lunge geschlossen werden. Die „Multiple breath 
inert gas washout“ (MBW) konnte zeigen, dass bei unauffälliger Lungenfunktion sich dort bereits signifikante Inhomogenitäten in der Ventilation finden.

Im Gegensatz zum allergischen Asthma bronchiale finden sich nicht erhöhte, sondern erniedrigte Stickoxid(NO)-Werte im Atemexhalat.

Die Pulsoximetrie zeigt, abgesehen von pulmonalen Exazerbationen, lange unauffällige Werte. Erste Auffälligkeiten finden sich bei körperlicher Belastung oder im Schlaf. Erst im fortgeschrittenen Stadium finden sich Auffälligkeiten in der Blutgasanalyse. Folgen der chronischen Hypoxie kann ein Cor pulmonale sein. Insofern sollte ein echokardiografisches Screening auf pulmonalen Hypertonus in regelmäßigen Abständen erfolgen.

Radiologische Diagnostik Ein konventionelles Röntgenbild des Thorax pro Jahr sollte als Verlaufsparameter durchgeführt werden. Erste Veränderungen sind Zeichen der Überblähung. Im Verlauf finden sich Ringschatten (Bronchialwandverdickungen und Bronchiektasen) und schleimgefüllte Bronchiektasen (• Abb. 158.3). Im Spätstadium der CF finden sich Zystenbildung, Fortschreiten der Bronchiektasen sowie Zeichen der pulmonalarteriellen Drucksteigerung. Zur vergleichenden Beurteilung wird der ChrispinNorman-Score des Röntgen-Thorax favorisiert. Hierbei wird nach Quadranten getrennt das Ausmaß von Fleckschatten, Überblähung, Bronchialwandverdickung und interstitiellen Streifen beurteilt. Die maximale Punktzahl beträgt 38. Eine gesunde Lunge würde mit 0 Punkten beschrieben, bei Kindern mit CF ist mit einer Zunahme von 1-2 Punkten pro Lebensjahr zu rechnen.

Sensitiver als konventionelle Röntgenaufnahmen, aber auch als die Lungenfunktion ist die High-Resolution-CT (HRCT). Hier lassen sich Bronchialwandverdickungen, Schleimpfropfen, Belüftungsinhomogenitäten und Bronchiektasen zu einem Zeitpunkt nachweisen, zu dem sie im konventionellen Röntgenbild nicht sichtbar sind (• Abb. 158.3). Komplementär im Informationsgehalt in Bezug auf entzündliche Veränderungen in der Lunge sind HRCT und Multiple breath inert gas washout (MBW).

Aufgrund der hohen Strahlenbelastung der HRCT gibt es Bestrebungen, gleich informative Magnetresonanzsequenzen zu entwickeln. Als Standard ist dieses bisher nicht etabliert.

\section{Therapie der wichtigsten Pathogene}

Pseudomonas aeruginosa $\mathrm{P}$. aeruginosa ist das wichtigste pulmonale Pathogen bei CF. 80 \% der Erwachsenen CF-Patienten sind mit P. aeruginosa infiziert, aber auch bei Säuglingen in einer australischen Geburtskohorte ließ sich schon bei $5 \%$ P. aeruginosa nachweisen.

Infektionen mit P. aeruginosa führen zu einer gesteigerten Morbidität, einem schnellerem Verlust an Lungenfunktion (ca. 2\%/Jahr) und ca. $90 \%$ dieser Patienten werden an den Folgen ihrer pulmonalen Pathologie sterben.

Durch den Nachweis von P.-aeruginosa-Klonen ist davon auszugehen, dass die Übertragung von Patient zu Patient eine wichtige Rolle spielt und nicht ein aus der Umwelt individuell erworbener Keim. Daher sind Mukoviszidosepatienten im ambulanten Bereich streng zu trennen, im stationären Bereich ist ein Einzelzimmer mit eigener sanitärer Versorgung zu fordern.

P. aeruginosa verfügt über adaptive Mechanismen, die es ihm ermöglichen, in den Atemwegen trotz Abwehrmechanismen des Wirtes und antibiotischer Therapie zu überleben. Durch den Switch zum Wachstum unter dem Schutz eines Biofilmes ist P. aeruginosa in der Lage, sich den inflammatorischen Abwehrmechanismen zu entziehen sowie eine antibiotische Therapie zu tolerieren.
Der Verlauf der Infektion lässt sich in 3 Phasen einteilen: Keine Infektion, intermittierende Infektion und chronische Infektion. Eine chronische Infektion liegt vor, wenn sich in $>50 \%$ der untersuchten mikrobiologischen Proben in den letzten 12 Monaten P. aeruginosa nachweisen lässt. Eine intermittierende Infektion liegt vor, wenn $<50 \%$ der Proben P.-aeruginosa-positiv waren.

In der Lunge wird die konduktive Zone (Trachea bis terminale Bronchiolen) mit anaerobem Sputum und die aerobe respiratorische Zone (respiratorische Bronchiolen bis zu den Alveolen) unterschieden. In der respiratorischen Zone finden sich keine Zilien, keine Becherzellen und keine submukösen Drüsen. Das Abwehrsystem besteht aus Alveolarmakrophagen und Defensinen. Das gesamte venöse Blut passiert die Alveloarkapillaren. Hier findet sich nur eine sehr dünne Barriere zwischen Luft und Blut. In der konduktiven Zone finden sich Zilien, Becherzellen und submuköse Drüsen. Inhalative Antibiotika erreichen die konduktive Zone, aber nur in sehr geringen Maß die respiratorische Zone. Systemisch (oral oder intravenös) verabreichte Antibiotika finden sich in hohem Maße in Lungengewebe, aber in geringem Maße im Sputum. Da P. aeruginosa zur Infektion der konduktiven wie der respiratorischen Zone führt, sollten Patienten von einer kombinierten Therapie aus systemischen und inhalativen Antibiotika profitieren.

Erst- und intermittierende Infektion Die deutsche Leitlinie empfiehlt für die frühe Eradikation eine Inhalation mit Tobramycin über 4 Wochen oder Ciprofloxacin p.o. kombiniert mit Colistin inhalativ über 3 Wochen. Eine zusätzliche Gabe von Ciprofloxacin zu einer Tobramycin-Inhalation führte zu keiner Verbesserung der Eradikationsrate.

Sollte eine Inhalation nicht möglich sein, ist eine intravenöse Inhalationstherapie in Betracht zu ziehen. Eine primär intravenöse Eradikationstherapie über 2 Wochen ist indiziert bei Patienten, bei denen im Rahmen des Erstnachweises eine, wenn auch milde, pulmonale Exazerbation besteht. Dieses gilt auch für Patienten mit Verschlechterung der Lungenfunktion.

Eine bildgebende Diagnostik sollte nach klinischen Gesichtspunkten (z. B. bei pulmonaler Exazerbation, Verdacht auf Pneumonie, Erguss, Pneumothorax) erfolgen.

Eine Kontrolle des Eradikationserfolges (frühestens 5 Tage nach Ende der antibiotischen Therapie, empfohlen sind auch 2-4 Wochen) erfolgt über eine Kultur von Proben aus den Atemwegen ([induziertes] Sputum, tiefer Rachenabstrich, bronchoalveoläre Lavage [BAL]). Wenn 3 konsekutive respiratorische Proben in einem Gesamtzeitraum von 6 Monaten negativ für P. aeruginosa sind, wird von einem Behandlungserfolg ausgegangen. Eine Kontrolle der P.-aeruginosaAntikörper ist 3 Monate nach erfolgreicher Eradikationstherapie und dann wieder einmal jährlich sinnvoll.

Sollte der Eradikationserfolg fehlgeschlagen sein, sind neben unten genannter intravenöser Antibiotikatherapie eine Therapie mit oralem Ciprofloxacin und mit inhalativem Colistin mit hoher Dosis (3-mal 2 Mio IE) über 3 Monate oder Tobramycin inhalativ (2-mal $300 \mathrm{mg} / \mathrm{Tag}$ ) über 4 Wochen empfohlen.

Intravenöse Antibiotikatherapie Bei mangelhafter Eradikation sollte eine intravenöse Antibiotikatherapie mit 2 Antibiotika aus verschiedenen Substanzgruppen (klassisch Drittgenerationscephalosporin Ceftazidim und Aminoglykosid Tobramycin) nach Antibiogramm erfolgen.

Die Therapiedauer liegt in der Regel bei 14 Tagen. Eine Therapiedauer von weniger als 10 Tagen birgt das Risiko der nicht ausreichenden Infektionsbekämpfung. Mehr als 21 Tage intravenöser Behandlung sind unbeliebt, da das Risiko einer allergischen Reaktion 
mit zunehmender Therapiedauer steigt. Aufgrund der höheren Clearance infolge von gesteigerter renaler und nonrenaler Elimination sind bei CF höhere Dosierungen (• Tab. 158.1) erforderlich.

Sofern eine chronische Infektion mit P. aeruginosa vorliegt, profitieren Patienten mit einem FEV1 $<70 \%$ von einer Dauertherapie mit intermittierenden Tobramycininhalationen (4 Wochen on/4 Wochen off). Für andere inhalative Therapien (Colistin, Gentamicin, Ceftazidim) ist die Evidenz nicht ausreichend. Tobramycin wie Colistin sind inzwischen auch als Trockeninhalat verfügbar. Vorteil ist die Zeitersparnis, aus der man sich eine bessere Compliance erhofft. Aztreonamlysinat hat sich in 3 Phase-III-Studien als sicheres und effizientes inhalatives Antibiotikum erwiesen, in der es einer Tobramycininhalation überlegen war.

Aufgrund der ototoxischen Potenz von Aminoglykosiden ist vor der ersten intravenösen oder inhalativen Anwendung eine audiologische Diagnostik (empfohlen: otoakustische Emissionen) durchzuführen.

Bei chronischer Besiedlung mit P. aeruginosa wird eine Gabe von Azithromycin (10 mg/kg KG/Tag; max. $500 \mathrm{mg} / \mathrm{Tag}$ ) jeweils an $3 \mathrm{Ta}-$ gen $(\mathrm{Mo} / \mathrm{Mi} / \mathrm{Fr})$ der Woche empfohlen. Hintergrund dieser Empfehlung ist die Verbesserung der Lungenfunktion durch Inhibition der bakteriellen Kommunikation („quorum sensing“) und Reduktion von Inflammation. Ein anderer positiver Aspekt ist die Reduktion pulmonaler Exazerbationen. Auch bei P.-aeruginosa-negativen $\mathrm{Pa}-$ tienten findet sich ein geringer Benefit durch eine AzithromycinDauertherapie.

Burkholderia cepacia Der Burkholderia-cepacia-Komplex umfasst 9 Spezies, u. a. B. cepacia genomvar I und III, B. multivorans, B. stabilis und B. vietnamiensis. Die Prävalenz in Deutschland beträgt 2,3\% bei Patienten $<18$ Jahre und 3,7 \% bei Patienten $>18$ Jahre. Die Übertragung zwischen Patienten ist nicht selten, daher ist auf strikte Trennung $\mathrm{zu}$ achten. Klinisch findet sich eine massive Verschlechterung der Lungenfunktion bei Bakteriämie. Aufgrund der Multiresistenz des Erregers ist die Eradikation oft nicht erfolgreich. Ziel der Therapie ist daher meist nur, die Zahl der Bakterien zu vermindern, erfolgversprechend scheint eine Dreifachtherapie mit Tobramycin, Meropenem und einem dritten Antibiotikum.

Methicillinresistente Staphylokokken Anders als vormals angenommen ist eine MRSA-Infektion mit einem schlechteren Verlauf assoziiert. Die Prävalenz liegt in Deutschland bei 9,9\%. Die Behandlungserfolge der Eradikationsversuche sind bei ansteigender Resistenz auf gängige Antibiotika (sofern sensibel, Rifampicin + Trimethoprim-Sulfmethoxazol, Linezolid, Vancomycin inhalativ) problematisch. Wichtig ist es, Hygienestandards diesbezüglich strikt einzuhalten bzw. die entsprechende Segregation.

Stenotrophomonas maltophilia Die Inzidenz liegt bei $12,4 \%$ in Deutschland. Eine große Kohortenstudie in den USA konnte nach Korrektur von Confoundern keinen Unterschied in Bezug auf den Verlust von Lungenfunktion bei positiven wie negativen finden. S. maltophilia scheint daher eher ein Marker für einen schweren Erkrankungsverlauf zu sein. Andererseits konnte eine kleinere Studie zeigen, dass eine chronische Besiedlung ein unabhängiger Risikofaktor für Exazerbationen ist. Bei plötzlicher Verschlechterung ist daher eine entsprechende Therapie zu diskutieren.

Nichttuberkulöse Mykobakterien Diese finden sich bei ca. 3,8$22,6 \%$ der CF-Patienten. CF-relevant sind M.-avium-Komplex, M. kansaii und M. abcessus. Zwei klinische Kriterien müssen erfüllt sein:
- Tab. 158.1 Dosierung und Applikation wichtiger Antibiotika zur Behandlung P.-aeruginosa-Infektionen bei CF

\begin{tabular}{|c|c|c|c|}
\hline Substanz & $\begin{array}{l}\text { Dosierung (mg/ } \\
\text { kg KG/Tag) } \\
\text { (Tagesmaximaldosis) }\end{array}$ & $\begin{array}{l}\text { Dosierungsin- } \\
\text { tervalle }\end{array}$ & $\begin{array}{l}\text { Anwen- } \\
\text { dung }\end{array}$ \\
\hline Amikacin & 30 & $12 \mathrm{~h}$ & i.v. \\
\hline \multirow[t]{4}{*}{ Aztreonam } & 150 & $6 \mathrm{~h}$ & i.v. \\
\hline & $(8 g)$ & & \\
\hline & 100 & kontinuierlich & i.v. \\
\hline & 225 (absolut) & $8 \mathrm{~h}$ & inhalativ \\
\hline \multirow[t]{2}{*}{ Cefipim } & $100-150$ & $8-12 h$ & i.v. \\
\hline & $(6 \mathrm{~g})$ & & \\
\hline \multirow[t]{3}{*}{ Ceftazidim } & $100-200$ & $8-12 h$ & i.v. \\
\hline & $(12 \mathrm{~g})$ & & \\
\hline & 150 & kontinuierlich & \\
\hline \multirow{2}{*}{$\begin{array}{l}\text { Ciproflo- } \\
\text { xacin }\end{array}$} & 40 & $12 \mathrm{~h}$ & p.o. \\
\hline & $(1,5 \mathrm{~g})$ & & \\
\hline \multirow[t]{3}{*}{ Colistin } & 2-6 Mio E/Tag & $8-12 h$ & inhalativ \\
\hline & $75.000 \mathrm{E} / \mathrm{kg} / \mathrm{Tag}$ & $8-12 h$ & i.v. \\
\hline & (max. 3-mal 2 Mio. E) & & \\
\hline \multirow[t]{2}{*}{ Imipenem } & $50-100$ & $6-8 h$ & i.v. \\
\hline & $(4 \mathrm{~g})$ & & \\
\hline \multirow{4}{*}{$\begin{array}{l}\text { Mero- } \\
\text { penem }\end{array}$} & $60-120$ & $8 \mathrm{~h}$ & i.v. \\
\hline & $(6 \mathrm{~g})$ & & \\
\hline & 60 & kontinuierlich & i.v. \\
\hline & $(3 \mathrm{~g})$ & & \\
\hline \multirow[t]{2}{*}{ Fosfomycin } & $300-400$ & $8 \mathrm{~h}$ & i.v. \\
\hline & $(15 \mathrm{~g})$ & & \\
\hline Netilmicin & 10 & $12 \mathrm{~h}$ & i.v. \\
\hline \multirow[t]{3}{*}{ Tobramycin } & 10 & $24 h$ & i.v. \\
\hline & $150-300 \mathrm{mg}$ (absolut) & $12-24 h$ & inhalativ \\
\hline & (600 mg) & & \\
\hline
\end{tabular}

a. Pulmonale Symptome oder noduläre/cavitäre Trübungen („opacity“) im Röntgen-Thorax oder HRCT mit multifokalen Bronchiektasen mit multiplen kleinen Knoten.

b. Andere Ursachen müssen ausgeschlossen sein.

Mindestens eines der mikrobiologischen Kriterien muss erfüllt sein:

a. 2 positive Sputumkulturen an 2 unterschiedlichen Zeitpunkten,

b. positive Kulturen aus mindestens einer bronchoalveolären Lavage,

c. Histopathologie vereinbar mit Mykobakterien in der Lungenbiopsie und positive Kultur aus Biopsie, Sputum oder Spülflüssigkeit.

Behandelt wird je nach Erreger und Sensibilität mit einem Makrolid (Clarithromycin, Azithromycin), Ethambutol, Rifampicin/Rifabutin und ggf. mit einem i.v.-Aminoglykosid. 
Staphylococcus aureus Eine Erstinfektion sollte über 2-4 Wochen mit einem entsprechend wirksamen Antibiotikum therapiert werden. Umgekehrt wird eher von einer kontinuierlichen Therapie gegen S. aureus abgeraten, da sich unter einem solchen Regime mehr P.-aeruginosa-Infizierte finden. Sofern die mikrobiologische Diagnostik eine Koinfektion mit P. aeruginosa und S. aureus zeigt und eine antibiotische Therapie gegen P. aeruginosa beabsichtigt ist, wird empfohlen, den S. aureus antibiotisch mit abzudecken.

Anaerobe Bakterien Anaerobe Bakterien (Prevotella, Veillonella, Propionibacterium und Actinomyces) können bei erwachsenen CF-Patienten (64\%) in hoher Zahl im Sputum isoliert werden. Die Besiedlung mit P. aeruginosa steigert die Wahrscheinlichkeit des Nachweises von anaeroben Bakterien im Sputum. In der BAL von Kindern fanden sich ähnliche anaerobe Bakterien. In der oben genannten Untersuchung waren alle Isolate sensibel auf Meronem. Sofern Anaerobier neben den primären Erregern signifikant zur Infektion und Inflammation beitragen, sollten sie antibiotisch mitbehandelt werden.

Virale Infektionen Die Prävalenz viraler Erreger (Respiratory syncytial virus [RSV], Influenzaviren, Adenovirus, Rhinovirus, Picornavirus und humanes Metapneumovirus) bei CF-Patienten und Gesunden unterscheidet sich nicht. Bei Säuglingen und Kleinkindern mit CF und viralen Infekten fand sich ein erhöhtes Hospitalisationsrisiko sowie eine verlängerte Zeit, bis der Verlust an Lungenfunktion wieder eingeholt werden konnte. Epidemiologische Studien ergaben eine zeitliche Koinzidenz zwischen der Erstinfektion mit P. aeruginosa und respiratorischen Virusinfekten. Pathophysiologisch lässt sich das durch die gesteigerte Adhärenz von bakteriellen Pathogenen an beschädigtem Epithel infolge viraler Infektion erklären. Ausreichende Evidenz, RSVImmunglobulin prophylaktisch zu verabreichen, gibt es nicht, aber eine Empfehlung für eine jährliche Influenzaimpfung.

Pilze Am häufigsten lassen sich Candida species sich bei CF nachweisen. Aussagen zur klinischen Relevanz einer langfristigen Kolonisierung sind derzeit nicht möglich.

Von den filamentösen Pilzen lässt sich am häufigsten Aspergillus fumigatus (57\%) nachweisen. Er findet sich selten bei jungen Kindern. In der Regel wird er in der Folge bakterieller Infektionen isoliert. A. fumigatus kann zur direkten Schädigung der Atemwegsschleimhaut führen und durch die Synthese u. a. von proteolytischen Enzymen die mukoziliäre Clearance behindern und zur Hemmung der Phagozytose führen. Aspergillome können (selten) in vorexistierenden pulmonalen Bronchiektasen auftreten und lassen sich in chronisch verlegten Nasennebenhöhlen finden. Bei wiederholtem Nachweis eines Aspergillus-Antigens sollte (mit entsprechender Empfindlichkeitsprüfung) antimykotisch behandelt werden. Bei transplantierten CF-Patienten kann es zu einer invasiven pulmonalen Aspergillose kommen.

Am zweithäufigsten findet sich unter den filamentösen Pilzen bei CF Scedosporium apispermium mit 8,6\%. In der Regel geht die chronische Kolonisierung nicht mit klinischen Symptomen einher.

Bei progressiver klinischer Verschlechterung sollte es aber als kausales Agens diskutiert und eine entsprechende antimykotische Therapie erwogen werden. Mittel der ersten Wahl ist Itraconazol, aufgrund der besseren Bioverfügbarkeit wird die orale Gabe favorisiert. Spiegelkontrollen sollten nach einer Woche erfolgen. Aufgrund der zunehmenden Resistenz gegen Itraconazol stehen als Alternativpräparate Voriconazol und Posaconazol zur Verfügung. Es kann aber auch zu einem klinischen Bild im Sinne einer allergischen bronchopulmonalen Aspergillose (ABPA) kommen (s. unten).
Exophalia dermatidis findet sich mit einer Prävalenz von 4,8$15,7 \%$ und führt in der Regel nicht zu klinischen Symptomen, anderseits kann der Pilz auch zu invasiven Infektionen führen.

Physiotherapie Physiotherapeutische Maßnahmen dienen der Sekretmobilisation und Unterstützung der Expektoration und sind einschließlich Sport- und Rehabilitationstherapie im Detail in den entsprechenden Beiträgen dargestellt. Die deutsche Leitlinie empfiehlt, möglichst früh nach Diagnosestellung der CF mit Physiotherapie und altersabhängig auch mit Sport zu beginnen.

\section{Inhalationstherapie}

$\beta 2$-Sympathomimetika Patienten mit bronchialer Hyperreagibilität profitieren von der Inhalation mit $\beta 2$-Sympathomimetika. Eine präventive Wirkung gegen Bronchospasmus bei Therapie mit inhalativen Antibiotika ist belegt. Für den chronischen Gebrauch findet sich in den amerikanischen Leitlinien keine Evidenz für oder gegen eine solche Empfehlung. Vergleichende Studien haben eine Überlegenheit des langwirkenden Salmeterol gegenüber dem kurzwirksamen Albuterol gezeigt.

Anticholinergika Ausreichende Evidenz für eine Verbesserung der Lungenfunktion durch Ipratropiumbromid findet sich bisher nicht. Für das langwirksame Tiotropiumbromid liegen noch keine Studien vor.

Dornase alpha Im Rahmen des Untergangs von Neutrophilen werden große Mengen Desoxyribonukleinsäure (DNA) freigesetzt. Dieses führt zu viskösem Sputum und zu Schleimpfropfen. Dornase alpha reduziert die Viskosität. Die Cochrane-Analyse konnte eine signifikante Verbesserung der Lungenfunktion und eine nichtsignifikante Reduktion von pulmonalen Exazerbationen zeigen. Die Inhalationstherapie mit Dornase alpha (1-mal 2,5 mg/Tag) wird bei Kindern $>6$ Jahre empfohlen.

Hypertone Kochsalzlösung Unter der Idee, dass hypertone Kochsalzlösung (6-7\%) die mukoziliäre Clearance steigert, wurden zahlreiche Studien initiert. Die Cochrane-Analyse konnte zeigen, dass eine Inhalation von 7\%iger Kochsalzlösung zu einer geringen FEV1-Verbesserung nach 4 Wochen führte, dieses war aber nach 48 -wöchiger Therapie nicht mehr nachweisbar. Auch wenn hypertone Kochsalzlösung im Gegensatz zu Dornase alpha keine dauerhafte Verbesserung der Lungenfunktion bewirkt, führt sie aber zu einer Verbesserung der Lebensqualität und zu einer Reduktion pulmonaler Exazerbationen.

Mannitol Mannitol-Trockenpulver (2-mal $400 \mathrm{mg} / \mathrm{Tag}$ ) führt $\mathrm{zu}$ einer signifikanten Verbesserung der Lungenfunktion (FEV1).

\section{Antiinflammatorische Therapie}

Ibuprofen Eine hochdosierte Gabe ( $25 \mathrm{mg} / \mathrm{kg} \mathrm{KG} \mathrm{2-mal/Tag)} \mathrm{führte}$ zu einem signifikant geringeren Abfall der FEV1 besonders bei jüngeren Patienten im Alter von 5-13 Jahren. Retrospektive Analysen konnten zeigen, dass viele Patienten aufgrund gastrointestinaler Beschwerden die dauerhafte Einnahme ablehnten. Wichtig ist es aufgrund der renalen Nebenwirkungen entsprechende individuelle pharmakokinetische Untersuchungen durchzuführen.

Nach den neuen amerikanischen Therapieleitlinien findet sich für Patienten im Alter von 6-17 Jahren eine Empfehlung aufgrund einer moderaten Verbesserung. Für Erwachsene reicht die Datenlage für eine Empfehlung nicht aus.

Orale Steroide Obwohl sich ein positiver Effekt auf die Lungenfunktion findet, wird für 6- bis 18-Jährige ohne Asthma oder ABPA (al- 
lergische bronchopulmonale Aspergillose) aufgrund der Nebenwirkungen von einer Dauertherapie abgeraten. Bei Erwachsenen lässt sich aufgrund der Datenlage keine Empfehlung dafür oder dagegen aussprechen.

Inhalative Steroide Studien evaluierten den Lungenfunktionsverlauf (gemessen an FEV1 und forcierter Vitalkapazität, FVC) und die Häufigkeit pulmonaler Exazerbationen nach Absetzen von inhalativen Steroiden in einer Kohorte von langzeitbehandelten Patienten. Es fanden sich keine signifikanten Unterschiede zwischen den verschiedenen Behandlungsarmen. Auch in anderen Studien kam es aufgrund oben genannter Untersuchungen zur Empfehlung der Ablehnung einer inhalativen Dauertherapie bei Patienten ohne Asthma oder ABPA. Kritisch sei angemerkt, dass so bei rezidivierenden Obstruktionen eine entsprechende Abklärung auf Asthma (signifikanter Broncholysetest, Hyperreagibilitätstestung) stattfinden muss. Ein nicht ausreichend antiinflammatorisch behandeltes Asthma bei CF ist sicherlich dem Erkrankungsverlauf nicht förderlich. Oben genannte Studie berücksichtigt nicht die Tatsache, dass in einer bestimmten vulnerablen Phase des Wachstums gewisse Stimuli (Infekte etc.) z. B. zum Asthma führen können. Insofern wäre eigentlich nur eine prospektive Geburtskohortenstudie geeignet, diese Fragestellung ausreichend zu beantworten.

Leukotrienrezeptorantagonisten Auch wenn die wenigen verfügbaren Studien zum Teil eine Verbesserung der Lungenfunktion zeigen, beurteilen die amerikanischen Richtlinien den Evidenzgrad nicht als ausreichend, um eine positive Empfehlung auszusprechen.

N-Acetylcystein Die amerikanischen Richtlinien beurteilen den Evidenzgrad nicht als ausreichend, eine positive Empfehlung auszusprechen. Keine der evaluierten Studien zeigte eine Wirkung.

Neue (kausale) Therapien

Ataluren (PTC 124) Nonsense- (prämature Stopcodon-) Mutationen in mRNA für CFTR (Typ-I-Mutationen) führen dazu, dass kein funktionstüchtiges CFTR synthetisiert wird. Ataluren ist ein oral verfügbares Medikament, welches eine ungestörte Translation ermöglicht. In einer Studie führte die Therapie zu einer (an nasaler transepithelialer Differenztestung) messbaren CFTR-Funktion bei ca. der Hälfte der Patienten bei guter Verträglichkeit.

Vertex 707 (Ivacaftor) Vertex 770 ist ein CFTR-Potenziator für Patienten mit mindestens einem G551D-Allel (Klasse-III-Mutation). Patienten erreichten in einer Studie unter oraler Therapie mit Vertex 770 nach 28 Tagen eine signifikante Reduktion der nasalen Potenzialdifferenz, des Chloridgehalts im Schweiß und einen FEV1-Anstieg um 8,7\%. Vertex 770 ist für Patienten mit oben genannter Mutation zugelassen. Über einen Zeitraum von 48 Wochen fand sich in einer weiteren Studie eine signifikante Verbesserung der Lungenfunktion (10,6\% FEV1). Unter Ivacaftor fanden sich signifikant weniger pulmonale Exazerbationen (55\%), eine Gewichtszunahme (2,7 kg) und eine Reduktion des Chloridgehalts im Schweiß $(-48,1 \mathrm{mmol})$. Aufgrund der substanziellen Verbesserung findet sich in den neuen amerikanischen Therapieleitlinien eine klare Empfehlung.

Denufosol Denufosol ist ein neuer Ionenkanalregulator unabhängig vom CFTR-Genotyp. Es steigert die Chloridsekretion durch einen kalziumaktivierten Chloridkanal, inhibiert die Natriumabsorption über den epithelialen Natriumkanal und steigert die ziliäre Schlagfrequenz. Dieses führt zu einem gesteigerten Flüssigkeitsangebot in den Atemwegen und einer verbesserten mukoziliären
Clearance durch eine durch Denufosol induzierte Aktivierung des P2Y2-Rezeptors auf den Atemwegsepithelien. In der TIGER1-Studie konnte eine signifikante Verbesserung der FEV1 unter Denufosolinhalation gezeigt werden. Aus bisher noch nicht geklärten Gründen konnten in der Folgestudie (TIGER 2) diese Ergebnisse nicht bestätigt werden.

Lancovutide (Moli 1901) Lancovutide ist ein polyzyklisches Peptid, welches die Befeuchtung der Atemwegssekrete über Aktivierung eines alternativen Chloridkanals verbessern soll. Studien konnten eine signifikante Verbesserung der Lungenfunktion (FEV1) zeigen.

\subsubsection{Komplikationen der Mukoviszidose}

\section{Allergische bronchopulmonale Aspergillose (ABPA)}

Diagnose Klassische Kriterien für die Diagnose einer ABPA sind (entsprechend den Kriterien der CF Foundation consensus Conference):

1. Akute oder subakute Verschlechterung bzw. Symptome (Husten, Giemen, Belastungsintoleranz, anstrengungsinduzierte Bronchialobstruktion, Verschlechterung der Lungenfunktion, gesteigerte Sputummenge), welche nicht einer anderen Ätiologie zuzuordnen sind.

2. Gesamt-IgE $>1000 \mathrm{IU} / \mathrm{ml}$ (sofern der Patient nicht mit systemischen Steroiden behandelt wird).

3. Positiver Pricktest auf Aspergillus fumigatus (Papel $>3 \mathrm{~mm}$ ) oder In-vitro-Nachweis von Serum-IgE-Antikörpern gegen A. fumigatus.

4. Präzipitierende Antikörper gegen A. fumigatus oder IgG-Antikörper gegen A. fumigatus.

5. Neue oder kürzlich zurückliegende Auffälligkeiten im Röntgen-Thorax (Infiltrate oder Schleimpfropfen) oder CT-Thorax (Bronchiektasen, „tree in bud“ etc.), welche auf Antibiotika oder Physiotherapie keine Besserung zeigen.

Ein Nachweis von A. fumigatus im Sputum ist nicht obligat.

Therapie Die Therapie der ABPA besteht in systemischen Steroiden (Prednisolon 1-2 mg/kg KG/Tag, max. $60 \mathrm{mg} / \mathrm{Tag}$ ). Diese führen zur Reduktion der inflammatorischen und immunologischen Aktivität, haben aber keinen Effekt auf die Antigenbelastung. Eine Reduktion der Pilzlast in den Atemwegen könnte die antigene Stimulation und die Inflammation reduzieren. Itraconazol scheint als Antimykotikum effizient und vermindert den Steroidbedarf.

Eine Alternative zu den nebenwirkungsbeladenen Steroiden ist der Anti-IgE-Antikörper Omalizumab.

\section{Hämoptysen}

Etwa 9,1\% der CF-Patienten leiden an Hämoptysen in einem 5-Jahres-Zeitraum. Eine massive Hämoptoe erleiden 4,1 \% der CF-Patienten in ihrem Leben, die durchschnittliche jährliche Inzidenz liegt bei $0,87 \%$ oder 1:115 Patienten/Jahr.

Therapie Die CF Pulmonary Guidelines unterscheiden zwischen geringer $(<5 \mathrm{ml})$, milder bis moderater $(5-240 \mathrm{ml})$ und massiver Hämoptyse $(>240 \mathrm{ml})$. Diese empfehlen bei geringer Hämoptyse noch keine Hospitalisierung, sofern sich keine Zeichen der pulmonalen Exazerbation zeigen, ist eine antibiotische Therapie nur bedingt notwendig.

Patienten mit massiven Hämoptysen sollten stationär behandelt werden. 
Bei milder Blutung sollte, sofern der Patient unter einer Therapie mit nichtsteroidalen Antiphlogistika (NSAID) steht, diese pausiert werden (aufgrund des Blutungsrisikos der NSAID durch den Effekt auf die Plättchenfunktion). Eine Embolisation der Bronchialarterien sollte bei instabilen Patienten mit massiver Hämoptoe erfolgen. Für eine Bronchoskopie vor der Intervention gibt es keine Empfehlung. Klare Empfehlungen, ob alle abnormen Gefäße embolisiert werden sollten oder nur jene, die verdächtig sind, existieren nicht. Gefahren der Embolisation liegen in der ungewollten Embolisation einer Spinalarterie. Biphasic positive airway pressure (BiPAP) sollte bei Patienten mit geringer Blutung weitergeführt werden, bei massiver Blutung sei eine Pause empfohlen. Eine Lungenresektion bei massiver Hämptyse bleibt Ultima Ratio.

Physiotherapie und Atemgymnastik sollte bei geringer Blutung weitergeführt werden, eine Pause wird bei massiver Hämoptyse empfohlen. Die Inhalationstherapie sollte bei leichter Hämoptyse weitergeführt werden, bei massiver Hämoptoe sollte die Inhalation von hypertoner Kochsalzlösung pausiert werden.

\section{Pneumothorax}

Die Inzidenz liegt bei 0,64\%. Etwa 3,4\% der CF-Patienten erleiden einen Pneumothorax in ihrem Leben. Die Größe des Pneumothorax wird als die Distanz zwischen Apex und Kuppel der Lunge bemessen. Es werden kleine $(\leq 3 \mathrm{~cm})$ und große $(>3 \mathrm{~cm})$ große Pneumothoraces unterschieden.

Therapie Die stationäre Behandlung wird bei großen Pneumothoraces empfohlen. Bei kleinen Pneumothoraces kann der Patient, sofern er klinisch stabil ist, ambulant geführt werden. Die Indikation für eine Drainage besteht bei einem großen und bei einem kleinen Pneumothorax, sofern der Patient klinisch instabil ist. Eine chirurgische Pleurodese wird bei rezidivierenden großen Pneumothoraces empfohlen. BiPAP sollte pausiert werden. Bis zu 2 Wochen nach Resolution des Pneumothorax sollte der Patient nicht fliegen, keine Spirometrie durchführen und nicht Lasten von mehr als $4,5 \mathrm{~kg}$ heben. Atemgymnastik wie PEP und intrapulmonale Perkussionsventilation sollten bei großen Pneumothorax in den meisten Fällen pausiert werden. Es besteht keine Indikation, die Inhalationstherapie zu pausieren.

\section{Lungentransplantation}

Die erste Lungentransplantation bei CF wurde 1983 durchgeführt. In Deutschland finden sich im CF-Register im Jahr 2010416 Patienten mit Lungentransplantationen, weltweit wurden zwischen 1984 und 20071155 Lungen bei Kindern mit CF transplantiert. Der Median der Überlebensdauer für Erwachsene nach Lungentransplantation liegt bei 6,4 Jahren.

Indikation Zwei Punkte sind wichtig im Hinblick auf den richtigen Zeitpunkt der Listung zur Transplantation. Zum einen sollte dieser so frühzeitig gewählt werden, dass eine Wartezeit überlebt wird und der Patient in einem so guten klinischen Zustand transplantiert wird, dass er die perioperative Periode überlebt und wirklich von der Transplantation profitiert. Entsprechend dem Toronto Lung Transplant Program sollten Patienten mit der Thematik konfrontiert werden, wenn die FEV1 unter $30 \%$ liegt oder rasant abfällt (besonders junge Frauen). Bei Kindern kann darüber diskutiert werden, wenn trotz aggressiver Therapie die FEV1 $<50 \%$ liegt. Weitere Kriterien sind ein Kohlendioxidpartialdruck $\left(\mathrm{pCO}_{2}\right)>50 \mathrm{mmHg}$, unterstützende Ernährung, intensivpflichtige pulmonale Exazerbationen, eine steigende Häufigkeit antibiotikapflichtiger Exazerbationen, rezidivierende Pneumothoraces, rezidivierende Hämoptysen (durch
Embolisation nicht kontrollierbar). Als weitere Indikation für die Lungentransplantation wird noch ein pulmonaler Hypertonus genannt. Zu berücksichtigten sind ferner eine zunehmende Sauerstoffbedürftigkeit und funktioneller Status (z. B. 6-Minuten-Gehtest).

Therapie Um die Funktion des Spenderorgans zu erhalten, ist eine konsequente immunsuppressive Therapie postoperativ erforderlich. In der Regel wird mit Kortison und einem weiteren Immunsuppressivum (z. B. Tacrolimus) behandelt.

Folgende Faktoren bestimmen die perioperative Mortalität: Primäre Graft-Dysfunktion (PGD) (Spenderorgan), Non-CMVInfektion, kardiovaskuläres Versagen und akute Abstoßung. PGD gilt als das wichtigste postoperative Problem. Diese tritt in der Regel innerhalb der ersten $24 \mathrm{~h}$ post OP auf und ist klinisch vergleichbar mit einem „acute respiratory distress syndrom (ARDS)“.

Komplikationen und Prognose Medizinische Komplikationen nach der Transplantation bestehen in einer Dysfunktion des Spenderorgans, einer Bronchiolitis obliterans zu gleichen Teilen bei Erwachsenen wie Kindern. Bei Kindern finden sich häufiger lymphoproliferative Erkrankungen, Wachstumsretardierung, chirurgische Komplikationen und Atemwegsinfektionen.

Maschinelle Beatmung gilt als relative Kontraindikation. Der negative Einfluss von B. cepacia auf die Prognose ist belegt. Auch ein gastroösophagealer Reflux gilt als Risikofaktor, gesichert ist der ätiologische Zusammenhang zwischen Reflux und Bronchiolitis obliterans. Diskutiert wird ein Zusammenhang zur akuten AbstoBungsreaktion.

\subsection{Zystische Fibrose im Magen-Darm-Trakt}

M. J. Lentze, J. Henker

\subsubsection{Exokrine Pankreasinsuffizienz}

Klinische Symptome Die exokrine Pankreasinsuffizienz bei CF zeigt bereits kurz nach der Geburt die typischen Symptome von massigen, fettglänzenden übelriechenden Stühlen als Folge der Maldigestion. Bei Nichterkennen der Gedeihstörung kann diese bis zur Atrophie führen. Das Längenwachstum ist lange normal. Im späteren Lebensalter bei schlechter Compliance oder schlechter Einstellung können Zustände von Vitaminmangel auftreten. Hier kann es zur Nachtblindheit infolge des Vitamin-A-Mangels und zu Augenmotilitätsstörungen und Gangstörungen aufgrund des Vitamin-E-Mangels kommen. Dabei handelt es sich um eine neuroaxonale Störung, die irreversibel ist im Gegensatz zur Nachtblindheit.

Diagnose Das Vorhandensein von fettglänzenden, übelriechenden Stühlen bei gleichzeitig pathologischem Schweißtest lässt kaum einen Zweifel an der exokrinen Pankreasinsuffizienz. Die bildgebende Diagnostik, insbesondere die Ultraschalluntersuchung, lässt in den ersten Lebensjahren im Stich. Manchmal kann ein etwas echodichtes Pankreas beobachtet werden. Einige CF-Patienten zeigen sonografisch - zum Teil auch schon pränatal - einen echoreichen Schichtungstyp der Darmschlingen durch eine Verdickung der Submukosa.

Nach wie vor ist die quantitative Bestimmung der Gesamtfettausscheidung im Stuhl durch eine Stuhlsammlung über 3-5 Tage die beste Methode zur Diagnose einer exokrinen Pankreasinsuffizienz. Wenn sie bei einer adäquaten Einfuhr mehr als $7 \mathrm{~g}$ Fett/Tag jenseits des Säuglingsalters übersteigt, liegt eine dekompensierte bzw. behandlungsbedürftige Pankreasinsuffizienz vor. Die Fettabsorption 
liegt bei Gesunden über $93 \%$ der aufgenommenen Menge. Patienten mit CF haben eine Fettausscheidung, die mehr als $10 \%$ der aufgenommen Menge beträgt. Aus der Fettaufnahme mit der Nahrung und der Stuhlfettausscheidung lässt sich der Fettresorptionskoeffizient berechnen nach der Formel:

\section{Fettresorptionskoeffizient $=$ \\ Fettaufnahme in $g-$ Fettausscheidung in $g$ Fettaufnahme in $g$}

Andere Pankreasfunktionstests wie die Bestimmung der fäkalen Elastase, der fäkalen Chymotrypsinaktivität, der Pankreolauryltest, ${ }^{13} \mathrm{C}$-Atemtest unter Verwendung verschiedener Tracer (1,3-Distearyl-2- ${ }^{13} \mathrm{C}$-Octanoyl-Glycerin, Cholesteryl- ${ }^{13} \mathrm{C}$-Octanoat, ${ }^{13} \mathrm{C}$-Tripalmitin, ${ }^{13} \mathrm{C}$-Hiolein, ${ }^{13} \mathrm{C}$-Trioctanoin, ${ }^{13} \mathrm{C}$-Triolein und ${ }^{13} \mathrm{C}$-Maisstärke) haben einen guten Aussagewert für die Feststellung einer exokrinen Pankreasinsuffizienz, erlauben aber nur bedingt eine quantitative Beurteilung der Pankreasinsuffizienz.

Therapie Liegt Pankreasinsuffizienz vor, so ist eine Ersatztherapie mit Enzympräparaten indiziert. Die Enzympräparate stammen fast ausschließlich aus Schweinepankreas. Das in Pulverform vorliegende Pankreatin wird entsprechend der Pharmacopoeia Europea (Ph. Eur.) standardisiert. Die höchste Effektivität besitzen säuregeschützte, mikrosphärische, darmlösliche Präparate (Mikrotabletten oder -pellets). Die Mikrotabletten oder -pellets werden im Magen aus der meist aus Gelatine bestehenden Kapsel freigegeben, vermischen sich mit dem Speisebrei und sollten zeitgleich mit diesem den Magen verlassen. Voraussetzung dafür ist, dass sie nur 1-2 mm, besser unter $1,4 \mathrm{~mm}$ klein sind. Erst oberhalb eines pH-Werts von 5,5 - also erst im Duodenum - löst sich der Säureschutz der Mikrotabletten oder -pellets. Bei eingeschränkter pankreatogener Bicarbonatsekretion ist diese Voraussetzung nicht immer gegeben. Dann sollte zeitlich begrenzt ein Magensäureblocker eingesetzt werden.

Die Dosierung von Pankreasenzympräparaten sollte sich nach dem Fettgehalt der Nahrung richten. Durch Ernährungsschulungen lernen Patienten und/oder Eltern, den Fettanteil von Mahlzeiten einzuschätzen. Außerdem sollten sie lernen, mit Nährwerttabellen zu arbeiten. Als Richtwerte für die Dosierung der Enzympräparate gelten 1000-2000 Lipaseeinheiten für $1 \mathrm{~g}$ Nahrungsfett bzw. 800010.000 Lipaseeinheiten/kg KG und Tag. Das Enzympräparat muss zur Mahlzeit eingenommen werden. Eine Ernährungsschulung ist mindestens einmal pro Jahr zu empfehlen.

Unter einer Enzymsubstitution kann die Ernährung energieund fettreich sein, so dass der Fettanteil bis zu $40 \%$ der Gesamtenergie ausmachen kann. Der Energiegehalt kann bis zu $130 \%$ der für Alter und Geschlecht empfohlenen Menge betragen.

Zeigt die Ernährung auch nach Steigerung der Enzymdosis nicht den gewünschten Erfolg (u. a. Gewichtsabnahme oder ungenügende Gewichtszunahme, anhaltend pathologische Stuhlentleerungen) sind ein 4-Tage-Ernährungsprotokoll zur Berechnung der Kalorienaufnahme sowie der Anteile von Fetten, Kohlenhydraten und Eiweißen zu erstellen und die Enzymtherapie sowie die PatientenCompliance (Bestimmung der fäkalen Chymotrypsinkonzentration) zu überprüfen. Ehe die Enzymtherapie deutlich erhöht wird, sollte eine 72-h-Fettbilanz durchgeführt und danach entschieden werden, ob ein Teil der Fette als MCT-Fette verabreicht wird. MCT sind Triglyceride mit Fettsäuren mittlerer Kettenlänge („medium chain triglycerides“, 6-10 C-Atome). MCT werden unabhängig von Gallen- säuren und Pankreaslipase im Darm resorbiert und ohne Bindung an Chylomikronen zur Leber transportiert.

Auch wenn die Gefahr einer fibrosierenden Kolonopathie durch extrem hohe Enzymdosen (>50.000 Lipaseeinheiten/kg KG/Tag) gering ist, sollten diese vermieden werden.

Bei der fibrosierenden Kolonopathie handelt es sich um eine nichtentzündliche Kolonobstruktion, die mit beträchtlicher intramuraler Fibrose des Kolons einhergeht. Gewöhnlich sind das Colon ascendens und transversum betroffen. Die betroffenen Patienten klagen über Symptome der Kolonobstruktion. Einige von ihnen mussten chirurgisch behandelt werden.

Etwa $20 \%$ der CF-Patienten haben eine exokrine Pankreassuffizienz mit Neigung zu rezidivierenden akuten Pankreatitiden.

\subsubsection{Pankreatogener Diabetes}

Bei einer schweren chronischen Erkrankung des exokrinen Pankreas, wie bei der Mukoviszidose, kann es zusätzlich zu einer endokrinen Pankreasinsuffizienz kommen, bei der der sog. pankreatogene Diabetes im Vordergrund steht. Je nach Alter sind 12-30\% der CFPatienten betroffen. Zur frühzeitigen Erfassung einer diabetischen Stoffwechsellage wird deshalb ab dem 10. Lebensjahr einmal pro Jahr ein oraler Glukosetoleranztest (oGTT) empfohlen.

\subsubsection{Hepatobiliäre Komplikationen}

Durch die hohe Expression des CFTR in den Gallenwegen ist bei Fehlfunktion desselben das hepatobiliäre System in mannigfacher Weise bei CF betroffen ( $\bullet$ Übersicht). Hierbei kann die Gallenblase betroffen sein mit dem Auftreten einer Mikrogallenblase und Atresie des Ductus cysticus, einer erweiterten Gallenblase und Gallensteinen. Daneben können auch die großen Gallengänge betroffen sein wie bei einer sklerosierenden Cholangitis. Die Leber selbst ist betroffen in Form einer fokalen oder multilobulären Zirrhose. Die fokale biliäre Zirrhose findet sich bereits bei $10-15 \%$ der CF-Kinder jünger als $1 \mathrm{Jahr}$, bei $20-50 \%$ aller CF-Patienten in der Kindheit und bei bis zu $72 \%$ der Erwachsenen mit CF. Die multilobuläre Zirrhose steigt von unter $1 \%$ bei Säuglingen und Kleinkindern auf $24 \%$ in Erwachsenen mit CF an. Es ist immer wieder darüber spekuliert worden, ob die Schwere der Lungenmanifestation mit der Lebermanifestation korreliert. Dies ist aber nach den heutigen Kenntnissen nicht der Fall. Eine besondere Manifestation der Leberkrankheit bei CF stellt die seltene, aber länger verlaufende neonatale Cholestase der Neugeborenen dar. In einigen Fällen verlief die Symptomatik entsprechend der einer extrahepatischen Gallengangsatresie mit acholischen Stühlen. Nur ganz wenige wiesen eine extrahepatische Gallengangsatresie auf, die meisten jedoch nicht. Aus diesem Grund muss bei jedem Kind mit Verdacht auf extrahepatische Gallengangsatresie eine CF mittels Schweißtest oder molekulargenetischer Untersuchung ausgeschlossen werden.

Komplikationen der Leber und Gallenwege bei zystischer

Fibrose

- Gallenblase:

- Mikrogallenblase

- Atresie des Ductus cysticus

- Vergrößerte Gallenblase

- Gallenwege:

- Gallengangssteine 
- Infantile Cholestase („inspissated bile syndrome“)

- Stenose des Ductus choledochus

- Sklerosierende Cholangitis

- Cholangiokarzinom

- Leber:

- Steatose der Leber

- Fokale biliäre Zirrhose

- Multilobuläre Zirrhose \pm portale Hypertension

Diagnose Die Diagnose der Hepatopathie bei CF ist schwierig. Die Ultraschalluntersuchung zusammen mit der Bestimmung der Leberfunktion in Blutuntersuchungen führt in der Regel weiter. Beim Ultraschall kann eine vermehrte Echogenität der Leber gefunden werden. Einfacher ist die Diagnose von Gallenblasen- oder Gallengangsanomalien. Gallensteine, Gallengangssteine werden in der Regel sicher entdeckt. Schwierig bleibt die Diagnose einer extrahepatischen Gallengangsatresie. Eine Mikrogallenblase, die sich in ihrer Größe nicht ändert, unabhängig davon, ob der Patient nüchtern ist oder nicht, gibt einen gewissen Hinweis. Auch die Gallengangsszintigrafie mit HIDA („hepatic iminodiacetic acid“) lässt im Einzelfall im Stich. Ist der Stuhl acholisch, findet sich auch eine pathologische Exkretion von HIDA in der Szintigrafie. Vor einer geplanten Laparotomie sollte ein Schweißtest, eine molekulargenetische Untersuchung oder ggf. eine Leberbiopsie zum Ausschluss anderer Erkrankungen durchgeführt werden.

Das Auftreten einer portalen Hypertension mit Ösophagusvarizen ist ein sicherer Hinweis auf die Entwicklung einer multilobulären biliären Zirrhose. Die Bestimmung der Standardleberwerte im Serum (Serum-Glutamat-Oxalacetat-Transaminase [SGOT], SerumGlutamat-Pyruvat-Transaminase [SGPT], $\gamma$-Glutamyl-Transferase $[\gamma-\mathrm{GT}]$, Gallensäuren) sowie Cholinesterase (CHE), Quick und Ammoniak als Parameter für die Entgiftungsfunktion geben im Langzeitverlauf einen Hinweis auf die Leberfunktionsstörung. Eine Leberpunktion ist unzuverlässig, da sie meist nicht repräsentativ für das ganze Lebergewebe ist. Sie kann sogar bei multilobulärer Zirrhose als normal befundet sein.

Therapie Die Behandlung der Leberfunktionsstörung bei CF ist schwierig. Neben der allgemeinen Therapie mit ausreichender Ernährung, Supplementierung von wasser- und fettlöslichen Vitaminen und der diuretischen Therapie bei Aszites hat sich die Gabe von Ursodesoxycholsäure etabliert. In einer Dosis von 15-20 mg/ $\mathrm{kg} \mathrm{KG/Tag} \mathrm{in} \mathrm{2-3} \mathrm{Einzeldosen} \mathrm{gegeben} \mathrm{hat} \mathrm{diese} \mathrm{Therapie} \mathrm{in} \mathrm{einigen}$ Studien eine Verbesserung der Leberfunktion gezeigt. Eine kürzlich durchgeführte Cochrane-Analyse hat allerdings gezeigt, dass die Routinegabe von Ursodesoxycholsäure bei CF mit Leberbeteiligung noch nicht gerechtfertigt ist.

Die Ösophagusvarizen werden ebenso behandelt wie die von Patienten ohne CF. Die Sklerosierung der Varizen bzw. ihre Gummibandligatur sind die Methode der Wahl. In seltenen Fällen ist eine portokavale Shuntoperation notwendig, in der Regel ein peripherer splenorenaler Warren-Shunt. Die Entscheidung hierzu muss im Gesamtbild der Schwere der Krankheit getroffen werden. Sie wird durch die Schwere des Lungenbefalls beeinflusst. Im Einzelfall kann die Shuntoperation die Zeit bis zu einer Lebertransplantation jahrelang überbrücken. Mit diesem Vorgehen kann der Patient stabilisiert werden bis zu einer Transplantation, auch bis zu einer evtl. kombinierten Lungen-Leber-Transplantation. Aber auch eine singuläre Lebertransplantation bzw. eine singuläre Lungentransplantation bei Hepatopathie ist möglich. Die Überlebenszeit der transplantierten Patienten, unabhängig davon, ob eine isolierte Lebertransplantation oder Kombination mit Lungen- und Lebertransplantation bzw. Herz-, Lungen- und Lebertransplantation durchgeführt wurde, beträgt derzeit $85 \%$ nach einem Jahr und $65 \%$ nach 5 Jahren.

\subsubsection{Intestinale Manifestationen}

Die intestinalen Manifestation der CF sind mannigfach und in der folgenden $>$ Übersicht zusammengefasst.

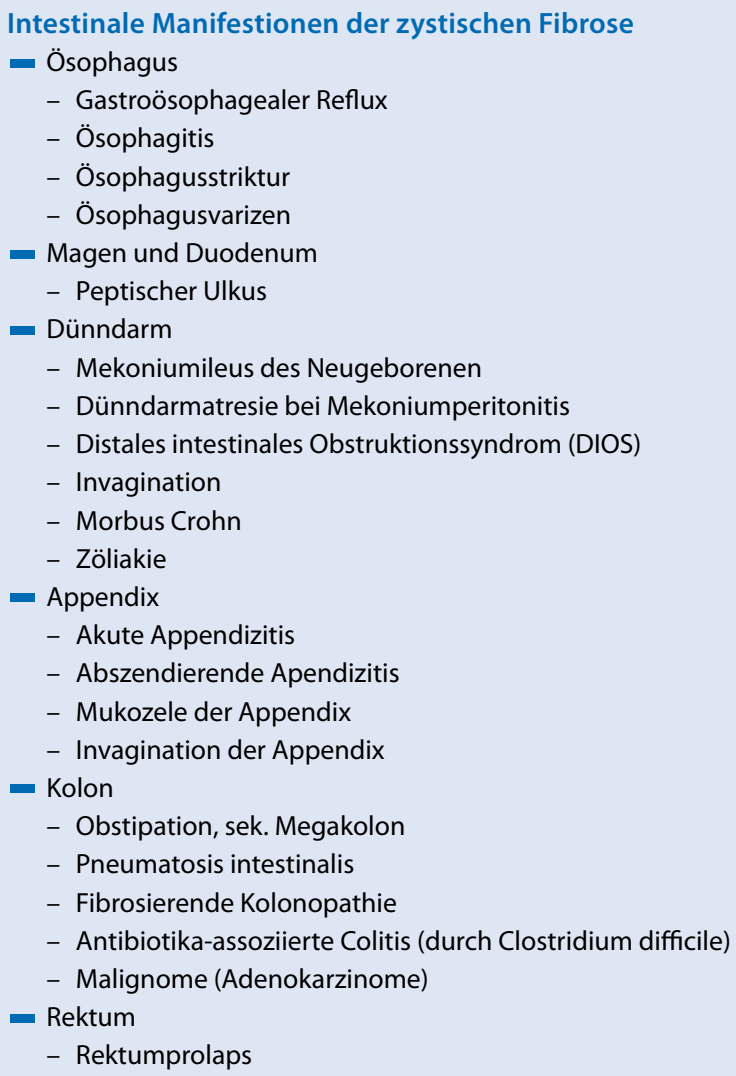

\section{Ösophagus und Magen}

Patienten mit CF leiden häufig unter einem gastroösophagealen Reflux (GÖR). Der GÖR wird mit einer Häufigkeit von $25 \%$ bei CF angegeben. Die Hälfte der Patienten mit GÖR entwickelt eine Ösophagitis. Der Reflux ist das Resultat der schweren Lungenerkrankung und ihrer Therapie mit Medikamenten, die den unteren Ösophagussphinkter relaxieren, des schweren Hustenreizes mit Kontraktion der Bauchmuskeln und des Zwerchfells sowie der Physiotherapie. Die Patienten mit GÖR klagen über Sodbrennen, Dysphagie und Regurgitation bzw. Gewichtsverlust oder Anorexie. Die Diagnostik entspricht der bei GÖR ohne CF: Durchführung einer Ösophagogastroduodenoskopie zur Diagnose einer Ösophagitis, einer Striktur oder einer Hiatushernie. Die Therapie beinhaltet die Gabe von Protonenpumpenhemmern (Omeprazol) für durchaus längere Zeit. Eine Fundoplicatio ist nur äußerst selten indiziert. Magen- und Duodenalulzera werden nur sehr selten gefunden. Auch ist eine Helicobacter-pylori-Gastritis bei Patienten mit CF selten. 


\section{Dünndarm}

Für die zwei wesentlichen Komplikationen der CF im Dünndarm, dem Mekoniumileus (MI) und dem DIOS besteht ähnlich wie bei der Pankreasinsuffizienz eine gewisse Genotyp-Phänotyp-Korrelation. Nur bei Patienten mit „schwereren“ Mutationen ( $\Delta$ F508/ $\Delta$ F508) und gleichzeitiger Pankreasinsuffizienz treten ein MI oder DIOS auf. Bei Patienten mit suffizienter Pankreasfunktion treten diese Komplikationen nicht auf. Der Zusammenhang ist wie bei der exokrinen Pankreasinsuffizienz in der mangelnden Funktion des CFTR zu suchen, der, sofern es den Dünndarm angeht, vermindert $\mathrm{Cl}^{-}$in das Lumen sezerniert. Dadurch kommt es zu Präzipitationen von Phospholipiden, vor allem Lecithin. Durch die eingenommenen Pankreasersatzpräparate wird das akkumulierte Lecithin zu Lysolecithin umgewandelt. Da aber die gleichen Extrakte keine Lysolecithinase enthalten, kommt es zu einer Anhäufung von Lysolecithin, welches durch seine hydrophobe Natur in der Lage ist, Epithelzellen zu zerstören. Eine gleichzeitig vorhandene abnorme Schleimproduktion, die exokrine Pankreasinsuffizienz, der verminderte Chloridtransport und eine herabgesetzte intestinale Motilität tragen zum Auftreten des Mekoniumileus und des DIOS bei.

\section{Mekoniumileus}

10-20\% aller Neugeborenen mit CF weisen einen Mekoniumileus auf, der durch eine Obstruktion des distalen Dünndarms infolge zähen Mekoniums im terminalen Ileums charakterisiert ist. Der Mekoniumileus tritt bereits in utero auf. In $10 \%$ aller Fälle mit Mekoniumileus kommt es bereits während der Schwangerschaft zu Perforationen des Dünndarms und zum Auftreten einer sterilen Mekoniumperitonitis, die radiologisch durch die charakteristischen Verkalkungen im Abdomen erkannt werden kann. Klinisch präsentieren sich die Neugeborenen durch das Fehlen von Mekoniumabgang in den ersten $48 \mathrm{~h}$ sowie die zunehmenden Zeichen des mechanischen Ileus im unteren Gastrointestinaltrakt. Eine Abdomenleeraufnahme zeigt die typischen Zeichen des Ileus (- Abb. 158.4). Der Mekoniumileus ist praktisch pathognomonisch für CF. Eine entsprechende Diagnostik mit Schweißtest und Molekulargenetik muss immer erfolgen. Therapie ist in der Regel die chirurgische Intervention mit Beseitigung des Ileus durch mechanisches Entleeren des Mekoniums. Manchmal muss eine vorübergehende Ileostomie angelegt werden.

\section{Distales intestinales Obstruktionssyndrom (DIOS)}

Nach der Neugeborenenperiode tritt bei älteren Patienten mit CF eine dem Mekoniumileus sehr ähnliche Krankheit auf mit distaler Obstruktion im terminalen Ileum und proximalen Kolon durch eingedickten Darminhalt. Früher wurde diese Symptomatik als Mekoniumileusäquivalent bezeichnet. Heute bezeichnen wir diese Symptomatik international als DIOS. Es tritt mit einer Häufigkeit von $5-7,5 \%$ in 1000 Patientenjahren in der Altersklasse der 15- bis 20-Jährigen auf und bis zu $35 \%$ in 1000 Patientenjahren in der Altersgruppe der 20- bis 25-Jährigen. Die Ätiologie ist unklar. Das DIOS tritt in der Regel nur bei Patienten mit exokriner Pankreasinsuffizienz auf.

Klinische Symptome und Diagnose Die Symptome sind schwere Bauchschmerzen in Form von Koliken, Erbrechen, selten auch Erbrechen von Galle, ein geblähtes Abdomen, das bei der Palpation eine peritoneale Abwehrspannung aufweisen kann. Im rechten unteren Quadranten tastet man verschiebliche Massen. Die Palpation ist schmerzhaft. Differenzialdiagnostisch ist an eine Appendizitis zu denken, die in einzelnen Fällen gleichzeitig zum DIOS vorkommen

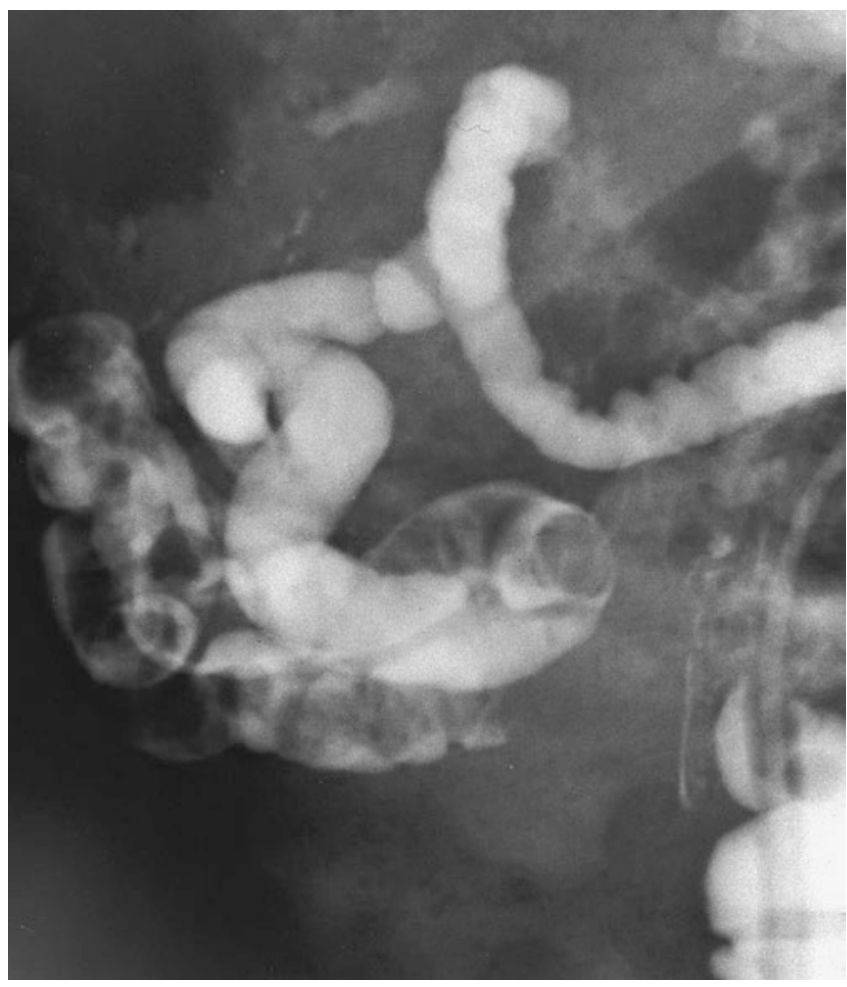

- Abb. 158.4 Mekoniumileus mit Darstellung von Stuhlmassen im terminalen Ileum eines Neugeborenen. Außerdem sind kalkspritzerartige Flecken im Abdomen als Folge der stattgefundenen Mekoniumperitonitis in utero sichtbar. (Mit freundl. Genehmigung von Dr. D. Emons, Radiologie des Zentrums für Kinderheilkunde Bonn)

kann. Ebenso kann eine gleichzeitige Invagination vorliegen. Die Diagnose kann in den meisten Fällen durch die Ultraschalluntersuchung gestellt werden. Hierbei ist die manchmal gleichzeitig vorhandene Invagination durch die typische Kokarde gut sichtbar zu machen. Eine Abdomenleeraufnahme weist ebenfalls auf die distale Obstruktion hin (• Abb. 158.5). Das DIOS ist häufig bei CF-Patienten nach einer Lungentransplantation.

Therapie Die Therapie des DIOS ist, soweit möglich, konservativ mit der oralen Gabe von großen Mengen von isoosmotischen Polyethylenglykollösungen (Golytely, Clean-Prep). Voraussetzung ist dabei allerdings der Ausschluss eines manifesten mechanischen Ileus oder einer Invagination. Hier kann eine vorsichtige rektale Spülung mit der gleichen Lösung versucht werden. Kommt es durch die Gabe von Polyethylenglykollösungen nicht zur Entleerung der Impaktionen, ist eine chirurgische Intervention anzustreben. Da sich das DIOS bei Patienten mit CF immer wiederholen kann, ist dies für die betroffenen Patienten eine schwere Beeinträchtigung ihrer Lebensqualität. Die kontinuierliche Einnahme von Polyethylenglykollösungen zu Hause vermindert die Zahl der DIOS und damit die stationäre Behandlung dieses Leidens im Krankenhaus. Bei rezidivierendem DIOS kann sogar an eine Kolon-PEG (perkutane endoskopische Gastrostomie) gedacht werden.

\section{Appendix}

Der Befall der Appendix bei CF kommt in verschiedenen Krankheitsentitäten vor. Sowohl eine akute Appendizitis wurde hierbei beschrieben als auch eine abszedierende Appendizitis, die purulente Mukozele der Appendix und die Invagination der Appendix mit rektaler Blutung. Die typischen Appendizitiszeichen im rechten 


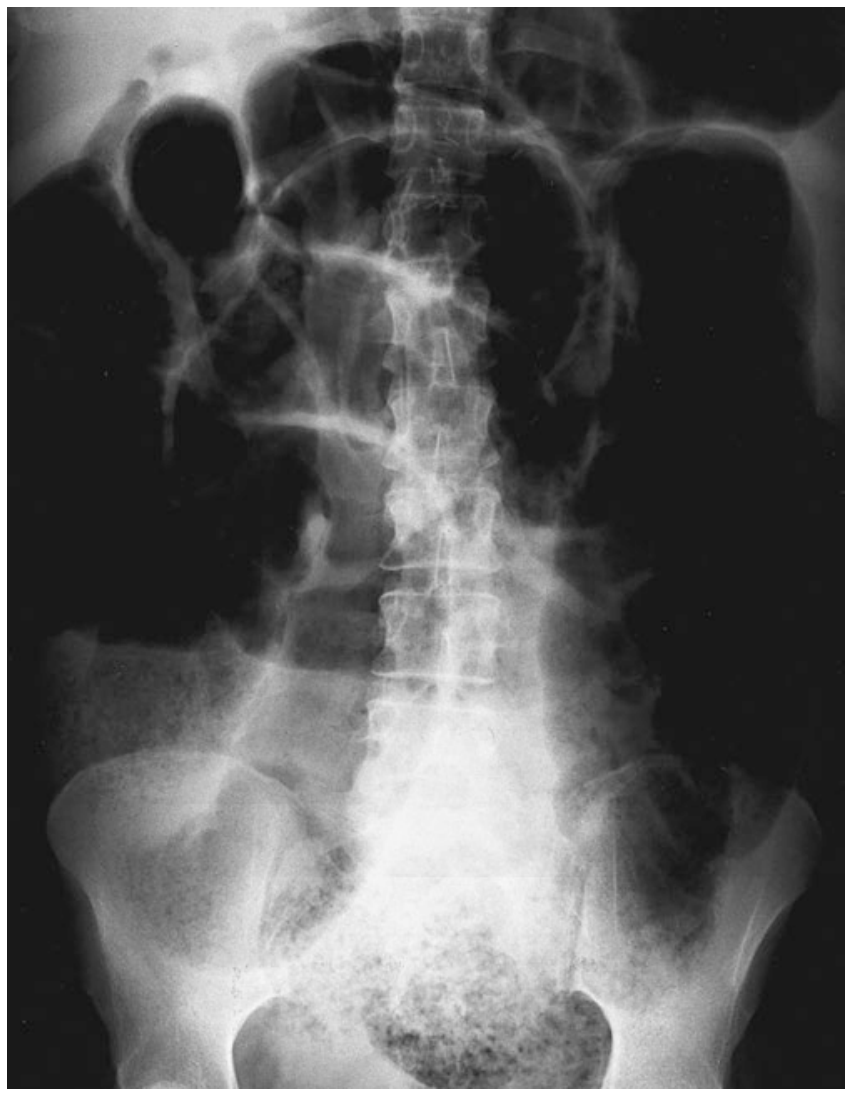

- Abb. 158.5 Abdomenleeraufnahme im Liegen bei einem erwachsenen Patienten mit CF und DIOS. neben den Zeichen eines distalen lleus mit stark geblähten Dünndarmschlingen sind die Stuhlimpaktionen im distalen Ileus und die fehlende Luft im Raum sichtbar

Unterbauch, eine Abwehrspannung als Zeichen der lokalen Peritonitis sollten an diese Möglichkeiten denken lassen. Diagnostisch kann eine Ultraschalluntersuchung weiterhelfen. Die chirurgische Intervention ist hier die Therapie der Wahl.

\section{Rektumprolaps}

Der Rektumprolaps kommt typischerweise bei CF-Kindern unter 5 Jahren vor und tritt häufiger bei Pankreasinsuffizienz auf. Er wird in der Regel vor der Diagnosestellung beobachtet. Die Ursache ist nicht vollkommen geklärt, sie wird dem starken Drücken bei der Defäkation von massigen Stühlen bzw. der Obstipation bei hohen Dosen von Pankreasersatzpräparaten zugeschrieben und auch dem ständigen Hustenreiz. Alle Kinder mit einem isolierten Rektumprolaps benötigen einen Schweißtest. Eine spezifische Therapie existiert nicht, in der Regel ist der Rektumprolaps selbstlimitierend durch die Behandlung der CF entweder durch die Substitution der Pankreasenzyme oder durch die Therapie der Obstipation mit Laxanzien (Laktulose).

\section{Clostridium-difficile-Kolitis}

Bei Adoleszenten oder Erwachsenen mit CF wird das Auftreten von schweren Kolitiden beobachtet, die mit schweren wässrigen Durchfällen, Bauchschmerzen und schlechtem Allgemeinzustand einhergehen. Das vermehrte Auftreten von Durchfällen wird anfänglich falsch als Versagen der Pankreasersatztherapie gedeutet. Erst die Darstellung des Kolons mit Ultraschall oder mittels CT des Abdomens zeigt dann ein monströs verdicktes Kolon im Sinne einer Pankolitis (• Abb. 158.6). In der Regel kann dann Clostridium difficile

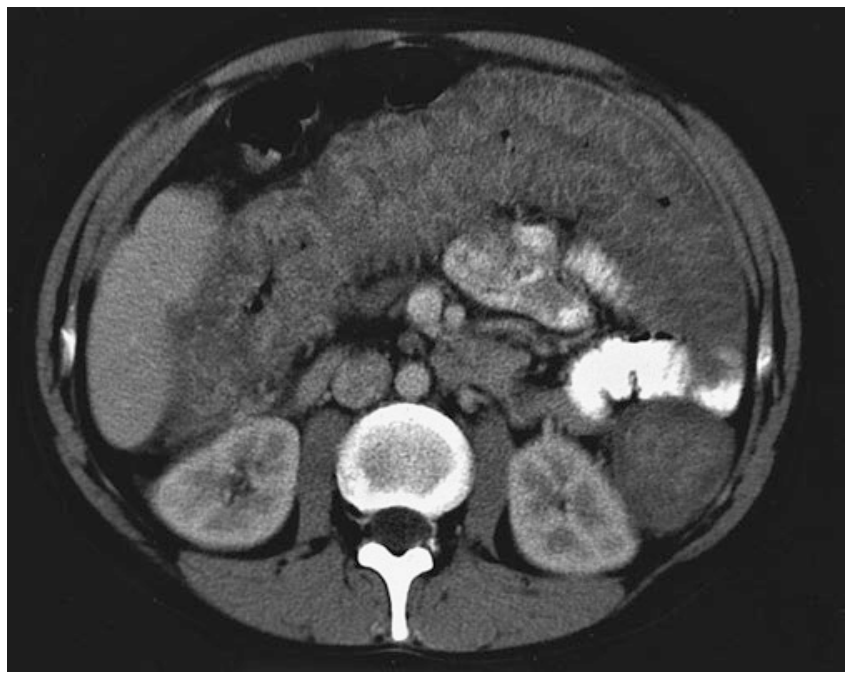

- Abb. 158.6 Das CT mit Kontrastmittel des Abdomens zeigt ein monströs verdicktes Kolon bei einem erwachsenen Patienten mit CF und Clostridium difficile. (Mit freundl. Genehmigung von Prof. Schild, Riadiologische Klinik)

im Stuhl nachgewiesen werden. Diese Form der Kolitis unterscheidet sich grundsätzlich von der pseudomembranösen Kolitis, die ebenfalls durch C. difficile hervorgerufen wird. Die Pankolitis verläuft sehr schwer, in einigen Fällen mit tödlichem Ausgang. Sie kann als antibiotika-assoziierte Kolitis interpretiert werden. Die Frage der Herkunft der C.-difficile-Bakterien ist unklar. Neben der nosokomialen Übertragung muss auch die asymptomatische Dauerausscheidung von CF-Patienten in Betracht gezogen werden. Die Therapie besteht in der intravenösen Gabe von Vancomycin und Metronidazol über längere Zeit (3-4 Wochen). Morphologisch bildet sich die schwere Kolitis nur langsam zurück. Verlaufskontrollen können mittels Ultraschall erfolgen. Die monströs verdickte Kolonwand bzw. ihre Rückbildung sind hierbei einfach zu beurteilen.

\section{Seltene andere Manifestationen im Gastrointestinaltrakt}

In erwachsenen Patienten mit CF wurden in den letzten Jahren Malignome im Gastrointestinaltrakt beobachtet. Hierbei wurden in der Mehrzahl der Fälle Adenokarzinome im Dünn- und Dickdarm beobachtet, aber auch Ösophagus-, Magen- und Pankreaskarzinome. Daher ist bei erwachsenen Patienten mit ungewöhnlichen gastrointestinalen Symptomen wie Anämie, rektalen Blutungen oder nicht DIOS-abhängigen Obstruktionen an die Möglichkeit einer malignen Entartung zu denken.

Infektionen mit Giardia lamblia wurden bei Patienten mit CF häufiger beobachtet als in der gesunden Normalbevölkerung. Bei $28 \%$ der Kinder mit CF und $44 \%$ der Erwachsenen konnten Giardia lamblia gefunden werden. Auch wurde der Morbus Crohn häufiger bei Patienten mit CF beobachtet als bei Gesunden. Die übliche Diagnostik mit oberer und unterer Endoskopie kann in diesen Fällen die Diagnose stellen. Insbesondere bei Symptomen wie Bauchschmerzen, Anämie, Hypoproteinämie und extraintestinalen Symptomen wie Arthritis sollte an das Vorliegen einer chronischentzündlichen Darmerkrankung gedacht werden. Selten wird neben der CF eine Zöliakie beobachtet. Bei Patienten, die trotz guter Einstellung mit Pankreasersatzpräparaten und adäquater kalorischer Ernährung weiterhin Symptome einer Malabsorption aufweisen, ist die Bestimmung der Anti-Gliadin-, Anti-Endomysium- oder AntiTransglutaminase-Antikörper hilfreich, bevor durch die Dünndarmbiopsie die Diagnose gestellt wird. 


\section{Literatur}

AWMF-Leitlinie (2013) S3-Leitlinie „Lungenerkrankung bei Mukoviszidose“. Modul 1: Diagnostik und Therapie nach dem ersten Nachweis von Pseudomonas aeruginosa

AWMF-Leitlinie (2013) S2-Konsensus-Leitlinie „Diagnose der Mukoviszidose (AWMF 026-023) unter Federführung der Gesellschaft für Pädiatrische Pneumologie

Borowitz DS, Grand RJ, Durie PR, The Consensus Committee (1995) Use of pancreatic enzyme supplements for patients with cystic fibrosis in the context of fibrosing colonopathy. J Pediat r 127:681-684

Flume PA et al (2007) Cystic fibrosis pulmonary guidelines. Chronic medications for maintenance of lung health. Am J Respir Crit Care Med 176:957-969

Flume PA et al (2009) Cystic fibrosis pulmonary guidelines. Treatment of pulmonary exacerbations. Am J Respir Crit Care Med 180:802-808

Flume $P$ et al (2010) Cystic fibrosis pulmonary guidelines. Hemoptysis and pneumothorax. Am J Respir Crit Care Med 182:298-306

Henker J (2012) Exokrine Pankreasinsuffizienz im Kindesalter. Ernährung Umsch 59:594-300

Kerem E, Corey M, Kerem BS et al (1990) The relation between genotype and phenotype in cystic fibrosis - analysis of the most common mutations. $\mathrm{N}$ Engl J Med 323:1517-1522

Lentze MJ (2001) Gastrointestinale Manifestationen der CF. Monatsschr Kinderheilkd 149:239-245

Mogayzel PJ et al (2013) Cystic fibrosis pulmonary guidelines. Chronic medications for maintenance of lung health. Am J Respir Crit Care Med 187:680689

O'Sullivan BP, Freedman SD (2009) Cystic fibrosis. Lancet 373:1891-1894

Ratjen FA (2009) Cystic fibrosis: Pathogenesis and future treatment strategies. Respir Care 54(5):595-602

Riordan JR, Rommens JM, Kerem B et al (1989) Identification of the cystic fibrosis: Cloning and characterization of complementary DNA. Science 245:1066-1072

Sinaasappel M, Stern M, Littlewood J et al (2002) Nutrition in patients with cystic fibrosis: A European consensus. J Cystic Fibrosis 1:51-75 